\title{
Performance Evaluation of Pre-processing Techniques on Diabetes Prediction
}

\author{
${ }^{* 1}$ Aminat B. Yusuf, ${ }^{2}$ Ogar O. Austin, ${ }^{3}$ Shinaigo Y. Tadi and ${ }^{3}$ Fatsuma Jauro \\ ${ }^{1}$ Department of Information and Communication Technology, Usmanu Danfodiyo University, Sokoto, Nigeria \\ ${ }^{2}$ Department of Computer Science, Federal Polytechnic, Kaltungo, Nigeria \\ ${ }^{3}$ Department of Computer Science, Ahmadu Bello University, Zaria, Nigeria \\ \{aminbolly l olomogarjnr | shinaigotadi I fatijauro\}@gmail.com
}

ORIGINAL RESEARCH ARTICLE

Received: 16-AUG-2021; Reviewed: 21-SEP-2021; Accepted: 22-DEC-2021

http://dx.doi.org/10.46792/fuoyejet.v6i4.686

\begin{abstract}
Medical industry contains a large amount of sensitive data that must be evaluated in order to get insight into records. The nonlinearity, non-normality, correlation structures and complicated diabetic medical records, on the other hand, makes accurate predictions difficult. The Pima Indian Diabetes dataset is one of them, owing to the dataset's imbalance, large number of missing values and difficulty in identifying highly risk factors. Some of these challenges have been solved using computational approaches such as machine learning methods, but they have not performed ideally, with pre-processing techniques being recognized as critical to achieving correct findings. The goal of this work is to apply multiple pre-processing approaches to increase the accuracy of some simple models. These multiple preprocessing techniques are median imputation in which null values are substituted by finding the median of the input variables dependent on whether or not the patient is diabetic and then follow by applying oversampling and under-sampling procedures on both majority and minority votes. These votes are applied in order to address the problem of class imbalance as pointed out from the literature. Finally, the dimension reduction Pearson correlation is used to detect high-risk features since it is effective at quantifying information between attributes and their labels. In this study, these techniques are applied in the same order to Linear Regression, Naive Bayes, Decision Tree, K Nearest Neighbour, Random Forest and Gaussian Boosting classifiers. The utility of the techniques on the mentioned classifiers is validated using performance measures such as Accuracy, Precision and Recall. The Random Forest Classifier is found to be the best-improved model, with 95 percent accuracy, 94.25 percent precision and 95.35 percent recall. Medical practitioners may find the provided strategies beneficial in improving the efficiency of diabetes analysis.
\end{abstract}

Keywords- Classifiers, diabetes, Pima Indian Diabetes dataset, pre-processing techniques

\section{INTRODUCTION}

$\mathrm{D}$ iabetes is a lifelong metabolic diseases that occurs when the body's cells are unable to utilize the insulin hormone or when the kidneys are unable to make enough insulin (American Diabetes Association, 2014). Type 1 , type 2 and gestational diabetes are the three types of diabetes (Danaei et al., 2011). Type 1 diabetes is more common in young people under the age of 30. Polyuria, thirst, constant hunger, weight loss, vision difficulties and exhaustion are the signs (Iancu et al., 2008). Type 2 diabetes, which affects the majority of individuals and causes insulin resistance in those over 45 years. It is caused by lifestyle, age and genetics. Laziness, weight loss, and fasting glucose levels $>7.0 \mathrm{mmol} / \mathrm{L}$ are all indications. Lastly, gestational diabetes which is a condition that affects pregnant women.

According to diabetes patient studies, there were 122 million adult (over 18 years old) with diabetes in 1980 and 422 million in 2014. This is an increase of $8.5 \%$ (Maniruzzaman et al., 2020). This figure is predicted to rise nearly 693 million by 2045(Cho et al., 2018). This statistic is alarming and immediate action is required in order to empower the medical system (Shafi \& Ansari, 2021).

\section{${ }^{*}$ Corresponding Author}

\section{Section B- ELECTRICAL/ COMPUTER ENGINEERING \& RELATED SCIENCES} Can be cited as:

Yusuf A.B., Austin O.O., Tadi S.Y. and Jauro F. (2021): Performance Evaluation of Pre-processing Techniques on Diabetes Prediction, FUOYE Journal of Engineering and Technology (FUOYEJET), 6(4), 341-346. http://dx.doi.org/10.46792/fuoyejet.v6i4.686
There is a wealth of medical data available on the internet and from other sources to aid in the prognosis of diabetic illness. However, diabetic medical data on the other hand is difficult to interpret. This is due to its nonlinear, nonnormal, correlation structured and complicated nature (Maniruzzaman et al., 2017). As a result, data comprehension and insight remain a big challenge. Machine learning is a fast-growing area with a variety of techniques that may be used to address these issues. These state-of-the-art techniques can aid in gaining insight into data and hence improve diabetes diagnostic when applied on machine learning algorithms.

This study investigates the impact of several preprocessing techniques on one of the most commonly used diabetes datasets, Pima India Diabetes (PID) dataset, in order to address some of the limitations of medical datasets mentioned above. According to (Kumari and Ahlawat, 2021; Mahedy et al., 2020), PID dataset is known to suffers from missing values and class imbalance, therefore dealing with these issues effectively is a major task. Thus, opens scope for further research to improve the accuracy.

As a result, the goal of this research is to improve classifiers performance using pre-processing techniques so that they can outperform existing diabetes prediction systems in terms of classification accuracy, precision and recall. The suggested approach employs the median imputation to fill in the missing values. This is because the datasets are biased to the left of the attribute distribution so rather than mean imputation this work employs median imputation. In order to remedy the imbalance dataset, the technicalities of synthetic minority oversampling and majority under-sampling procedures 
were used. To further boost classification accuracy and minimize the overall complexity of the prediction system, this study uses the Pearson Correlation Feature Selection approach to choose the most important features selected based on their coefficient values of correlation. Besides Pearson Correlation algorithm is good at measuring information between attributes and attributes with labels(Daru et al., 2021) and handling numeric attributes efficiently(Schober et al., 2018). The proposed approach was predicted using simple classification methods such as Naive Bayes (NB), Decision Tree (DT), Logistic Regression (LR), K-Nearest Neighbour (KNN), Random Forest (RF) and Gradient Boost (GB). The performance of these algorithms was assessed using precision, accuracy and recall.

\section{RELATED WORK}

The challenge of enhancing the accuracy of the classifier on the PID dataset is a prevalent one. Several approaches for addressing missing values, rebalancing the dataset and feature selection algorithms to minimize the feature set have been presented in the literature to make the classifier quicker and more accurate. This section gives a quick summary and sets the stage for the suggested work.

Jeatrakul et al. (2010) proposed a combination of synthetic minority oversampling and the Complementary Neural Network (CMTNN) to handle imbalanced data on several datasets along with PID. Artificial Neural Network, SVM and KNN were used to demonstrate the efficiency of the proposed balancing method. Boughorbel et al. (2017) proved the inadequacy of resampling techniques when number of samples in the small class is limited. 64 datasets, including PID datasets, were classified using an optimum Bayesian classifier. As a result, there was a higher level of consistency and precision in dealing with the skewed data. Vaishali et al. (2017) suggested a prediction model for PID dataset. Goldberg's Genetic algorithm was tested on the NB, Multilayer Perceptron (MLP), DT, and Multi Objective Evolutionary Fuzzy Classifiers. The proposed method was applied to the incomplete and imbalanced dataset. The classifier accuracy show improvement for all the classifiers.

Kalaiselvi \& Sujarani (2018) proposed method to improve PID data. The problem of missing values was solved by omitting them from the dataset. While selection uses Correlation Feature Selection and a Probabilistic Neural Network (PNN) classifier was used to train and test the suggested model. The accuracy of $95.31 \%$ was reported. Ogedengbe \& Egbunu (2020) exploited Classifier Subset Evaluator selection method on DT classifier using WEKA. The work fails to address missing and imbalance nature of the dataset. The experimental result showed the supremacy of the DT over the traditional classifiers. Mahedy et al. (2020) implemented Mutual Information based selection strategy. Missing values were addressed by using the mean imputation and oversampling method for handling the imbalanced data. The authors enhance the accuracy of Tree-Based algorithms DT, RF and Extra Tree classifier. Nnamoko \& Korkontzelos (2020) propose a selective data pre-processing approach to tackle outliers and imbalanced. The methods employ inter quartile range algorithm and synthetic minority oversampling technique. The performance was tested on Adaboost, RF, NB, SVM with radial basis function and C4.5 classifiers. C4.5 classifier obtained the highest performance. Sreejith et al. (2020) offered a methodology that addresses the feature selection difficulty and class imbalance. The dataset was balanced at the data level using enhanced synthetic minority over-sampling technique. The work used Chaotic Multi-Verse Optimization as selection method. The PID dataset was was experimented on RF, with a result of $0.89 \mathrm{~F} 1$ and $89.04 \%$ accuracy. Kumari \& Ahlawat (2021) proposed diabetes model for PID dataset. The work devises Z-score to exclude outliers, fill missing data with regression method and the Pearson correlation methodology for feature selection. The technique achieves classification accuracy of $89.82 \%$ on KNN using default parameter settings of $\mathrm{k}$. Azad et al. (2021) proposed pre-processing layer that is responsible for handling missing values, detection of outlier and selecting significant features. They ignored the missing entries, resolve outlier using synthetic minority oversampling technique and feature selection using both genetics algorithm and correlation. This was evaluated on decision tree classifier with an accuracy of $82.1256 \%$.

\section{Methodology}

Data pre-processing and feature selection methods affect the performance of any classification algorithm. As a result, our research attempted to manage the data as efficiently as possible. Our research objective is to deploy multiple pre-processing techniques and feature selection method, thus investigate their impact on PID dataset. The framework of this study has depicted in Fig 1 shows steps taken in achieving these.

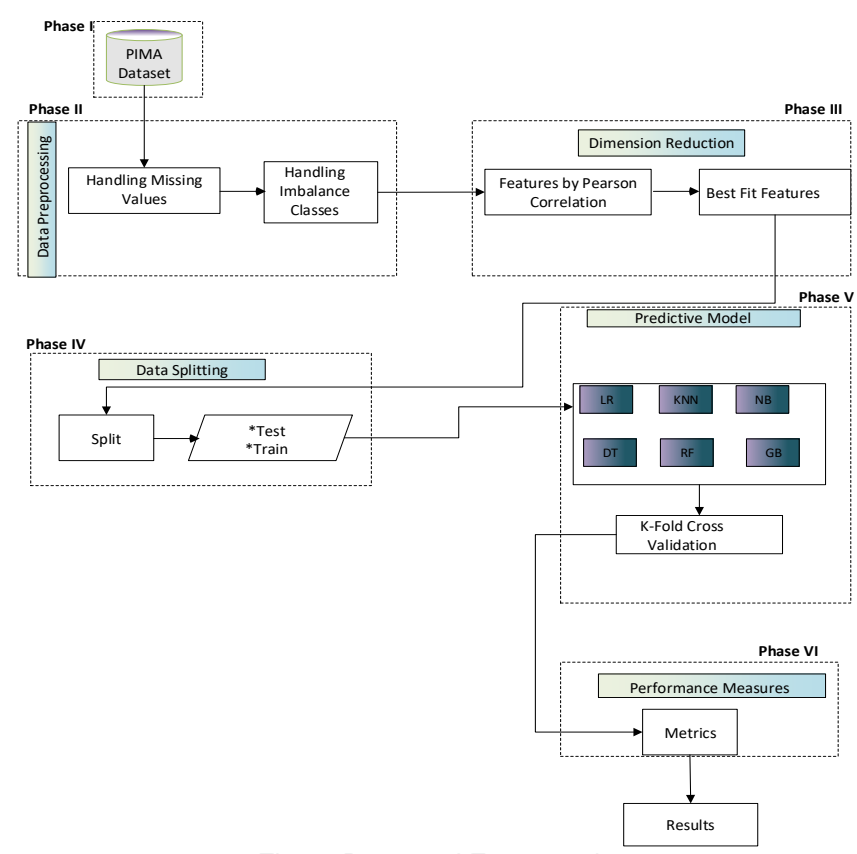

Fig. 1: Proposed Framework

\subsection{DATASET}

The proposed methodology is tested using the PID diabetes dataset from the Pima Indian population, which may be found on the University of California's website (Blake and Merz, 1998). Categorical variables are the 
target attribute with 0 and 1 values representing tested negative and positive for diabetes, respectively. There are 768 diabetes patients, including 268 diabetic patients (positive) and 500 non-diabetic patients (negative).

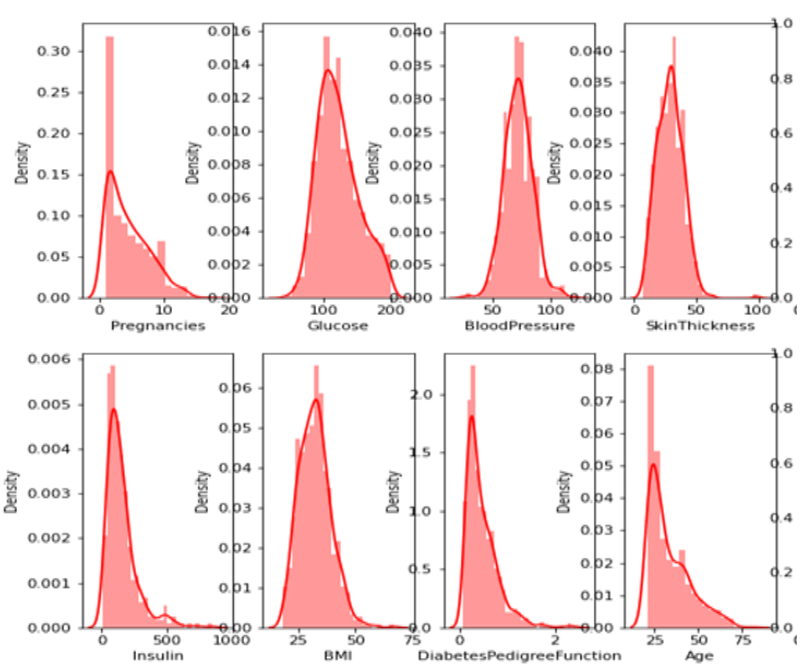

Fig. 2: PID dataset Distribution

In addition, to acquire a better understanding of the dataset, the distribution properties of all the attributes were computed. As seen in fig 2, the majority of the traits are skewed to the left.

\subsection{DATA PRE-PRocessing}

The pre-processing stage involves dealing with missing values/ null values and data imbalance that may be present in the dataset. These are briefly explained as follows:

\section{i) Handling missing values}

The variables in PID dataset were analysed for the presence or absence of missing or null values, which might cause classifiers to make incorrect predictions. The null values found in the dataset are shown in Figure 3 where the null values and their number of counts can be found as follows: Pregnancies: 11, Glucose: 5, Blood Pressure: 35, Skin Thickness: 227, Insulin: 374, and Body Mass Index: 11.

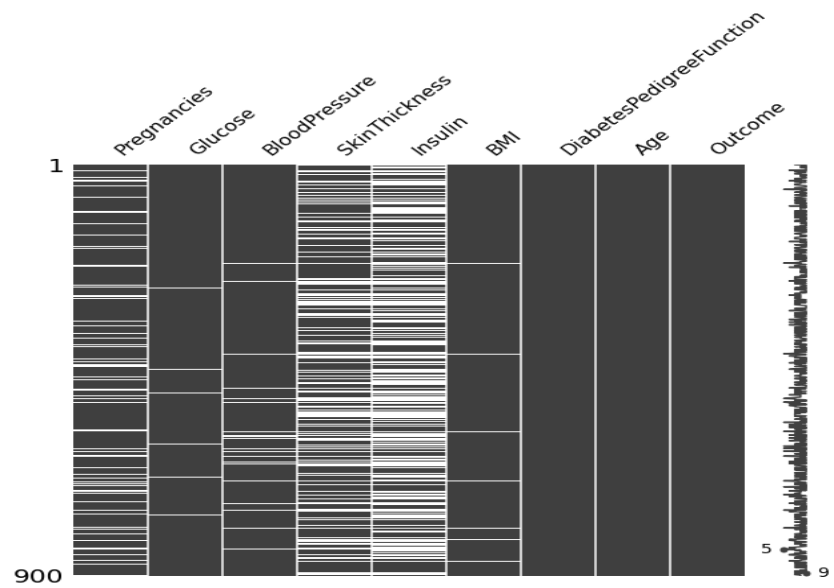

Fig. 3: Pictorial representation of null values in the PID dataset

Instead of dropping, the null values in their respective entries the values were filled using median imputation, which can be formulated as in Equation (1). The median imputation is advantageous since major of the attributes are skewed to the left and it imputes continuous data without introducing outliers. Where $\mathrm{x}$ is the instances of the feature vector that lies in $n$-dimensional space.

$Q(x)=\left\{\begin{array}{cc}\operatorname{median}(x), & \text { if } x \text { is missed } \text { or null values } \\ x, & \text { otherwise }\end{array}\right.$

\section{ii) Handling imbalance data}

In medicine, both oversampling and under sampling approaches were employed to cope with unbalanced data (Blagus and Lusa, 2015). The PID dataset is unbalanced because the negative class accounts for 65.1 percent of the class value, while the positive class accounts for only 34.9 percent of the class value. Because of the unequal distribution of wealth, the majority class is prejudiced against the minority. In order to tackle these challenges, random over sampler and under sampler techniques have been used. It reduces random synthetic data for the majority class from its nearest neighbours using Euclidean distance and generates random synthetic data for the minority class. This is to decrease or raise the number of data instances. The minority training set (268 diabetes patients) is produced using oversampling approaches, whereas the majority training set (500 nondiabetic patients) is decreased using under-sampling strategies in this work. The total number of occurrences in the data set was raised from 768 to 900 .

\subsection{Dimension Reduction}

Dimension reduction is a data mapping technique that reduced dimensional space by eliminating unnecessary data variance (Tsang et al., 2006). Feature selection is one of the forms in machine learning as its saves time, cost, reduces over-fitting and improves classification accuracy. Filters, wrappers and embedding methods are some of the most widely utilized feature selection strategies.

The Pearson correlation feature selection a filtering technique was employed in this study to determine the major risk factor for diabetes illness. The algorithm is good at measuring information between attributes and attributes with labels (Daru et al., 2021) and handling numeric attributes efficiently (Schober et al., 2018). It employs a mathematical function to figure out how the independent and dependent variables are related. The characteristics are chosen based on the correlation coefficients' values. The most predictive feature with the class variable is deemed significantly related and is included in the final feature set. For instance, consider the dataset D, which contains set of features F as in (2).

$$
F=\left\{x_{1}, x_{2}, x_{3} \ldots, x_{n}\right\}
$$

The classes $C$ of values $\mathrm{c}$ and the set are treated as random variables, as defined with the Pearson's linear correlation coefficient in (3). ( $r)= \pm 1$ if $X$ and $Y$ are linearly linked, and $(r)=0$ if they are completely uncorrelated.

$$
r=\frac{\sum_{i=1}^{n}\left(x_{i}-x\right)^{2}\left(c_{i}-c\right)}{\sqrt{\left[\sum_{i=1}^{n}\left(x_{i}-x\right)^{2}\right]\left[\sum_{i=1}^{n}\left(c_{i}-c\right)^{2}\right]}}
$$




\section{Predictive Model}

Six machine-learning classifiers were employed in this study i.e., Logistic Regression (LR), Naive Bayes (NB), KNearest Neighbour (KNN), Decision Tree (DT), Random Forest (RF) and Gradient Boost (GB). In order to improve the performance of KNN (Kumari and Ahlawat, 2021), DT (Mahedy et al., 2020; Ogedengbe and Egbunu, 2020), NB (Mahedy et al., 2020) and some unexplored classifiers such as LR, RF and GB were tested on the PID dataset. The models were trained on extracted features as identified by pre-processing (see Section 3.2) and dimension reduction (see Section 3.3) techniques to predict the presence of diabetes. The classifiers are discussed briefly in subsequent subsections.

\subsection{LINEAR REGRESSION}

LR a basic supervised learning approach for predicting the link between explanatory factors and dependent variables was validated on the data. PID data has only two dependent outcomes i.e., a binary variable 0 and 1 through fitting a linear equation of equation (4) (Kumari et al., 2020) to experience data, the goal of logistic regression is to find the model that best represents the relationship between a set of independent explanatory factors and the binary variables.

$$
y=\beta_{0}+\beta_{1} x_{1}+e
$$

Where $y$ is the response variable, the model coefficients error (e) and the model coefficients: $\beta 0$ and $\beta 1$. These unknown constant values indicate the intercept and slope, which are learned during the training phase.

The application of LR using Sciklearn libraries, the study applied parameter tuning gridsearchcv() method on solver and maximum number of iterations. The solver is the algorithm utilized to solve the optimization issue, the options are "newton-cg," "sag," "saga," "liblinear" and "lbfgs". Also, for the solvers to converge it was set between 100 and 500 iterations. This experiment revealed the LR achieved the highest accuracy when the solver was set to "lbfgs" at the maximum iteration of 100 while other parameters left at their default settings.

\subsection{NAÏVE BAYES}

NB is used to validate the data. The basic assumption of NB is that features are mutually independent (Cover, 1965) as in Equation (5).

$$
P(\text { class } \mid \text { data })=\frac{P(\text { data } \mid \text { class }) * P(\text { class })}{P(\text { data })}
$$

Where $\mathrm{P}$ (class I data) is the probability of the given class in the data. The two NB parameters priors and Var smoothing were used in this study due to their convenience of use and excellent performance in previous study (Yusuf and Kiyea, 2021). Priors represent the prior probability of the classes. The prior probability will not be justified by the data if this parameter is supplied while fitting the data, as it was in this inquiry. In this experiment, the NB achieved maximum accuracy by setting var smoothing to 0.66 and leaving the other parameters as their default.

\subsection{K-NEAREST NEIGHBOUR}

$\mathrm{KNN}$ is third classifier used to validate the data. It uses the majority of its K neighbours to classify each unknown occurrence in the training set. The value of $\mathrm{K}$ neighbours has a major impact on the classifier's efficiency. A higher $K$ value causes greater bias, whereas a low number causes it to be more sensitive to noise. In this work, value of $\mathrm{K}$ is determined using the Sciklearn libraries' default settings. The KNN achieved accuracy with a value of $\mathrm{K}$ of 5 , Euclidean distance and uniform weight function.

\subsection{DECISION TREE}

DT is the fourth non-parametric supervised learning strategy applied in this study. Since the target class is categorical, then the tree is right choice. Decision tree has root node at the top, internal nodes that represent attributes, while the branch represents a decision rule and each leaf node provides the conclusion in a flowchart-like tree structure. The decision rule is made using the best rate of splitting criteria where features with the best remain in the tree.

The criterion used for splitting are implemented on gridsearchcv that optimizes the maximum depth, the range of features to check while looking for the optimal split, the minimum number of samples required dividing an internal node and the criterion: the Gini-Index and entropy were used. This experiment revealed that DT achieved the highest accuracy when the minimum number of samples split was 40 , maximum depth of 8 , criteria of gini, and the maximum features of sqrt.

\subsection{RANDOM FOREST}

$\mathrm{RF}$ is the fifth multi-decision tree ensemble classifier applied. It divides the data set into sub-samples and then uses average to fit a number of decision tree classifiers to the sub-samples. The most important hyper-parameters tuned in this study is similar to the DT classifier discussed in 4.4 .

\subsection{GRADIENT Boosting}

GB is the sixth classifiers applied in this study. An increase loss function, a weak learner to produce predictions, and an additive model to combine weak learners to minimize the loss function are the three main components of GB. The application of gradient tree Boosting as implemented on python is handled as a simple classifier rather than combine with any of the weaker learning classifiers. This is to test how good is GB in predicting on its own. This study left the default parameters setting as given in sciklearn libraries.

\section{RESULT AND Discussion}

Six machine learning algorithms were employed in this research: LR, NB, KNN, DT, RF, and GB. As mentioned in subsection 3.2, all of these methods were used on the PID dataset.

\subsection{EVALUATION AND VALIDATION}

The algorithms in this research were implemented using the discussed parameters under each of the subsection 4 . PID dataset was split in a 4:1 in respect of training and testing datasets. The intention of partitioning the data is to prevent the model from overfitting during model testing when applied on test dataset. Furthermore, the dataset was divided into $K$ folds where every $(k-1)$ 
fraction of each iteration is utilized for training, while the remainder is used for testing and the method is repeated for k-iterations. Several scholars used different values of $\mathrm{k}$ for $\mathrm{k}$-fold cross validation. In this study, the value $\mathrm{k}=10$ is used since it produces good outcomes in the experiments and following the same approach with (Mahedy et al., 2020).

Table 1. Performance measure

\begin{tabular}{ccc}
\multicolumn{3}{c}{ Table 1. Performance measure } \\
\hline Metrics & Definitions & Formula \\
\hline $\begin{array}{c}\text { Accuracy } \\
\text { (ACC) }\end{array}$ & $\begin{array}{c}\text { the ratio of correct } \\
\text { predictions to total } \\
\text { predictions } \\
\text { the number of } \\
\text { accurately }\end{array}$ & $A C C=\frac{T P+T N}{T P+T N+F P+F N}$ \\
Precision & $\begin{array}{c}\text { recognized positive } \\
\text { (PR) } \\
\text { the total number of } \\
\text { positive samples } \\
\text { recall is a metric that } \\
\text { tests how well a } \\
\text { model can detect } \\
\text { positive samples }\end{array}$ & $P R=\frac{T P}{T P+F P}$ \\
& & $R=\frac{T P}{T P+F N}$ \\
\hline
\end{tabular}

Finally, the algorithms were assessed for validity using the confusion matrix on three distinct metrics: accuracy, precision and recall. Table 1 displays the confusion matrixderived metrics for each of the measures utilized. Represented by TP (True Positives) that stands for actual diabetes and predicted diabetes, FN (False Negatives) means actual diabetes but predicted to not diabetes, FP(False Positives) means predicted diabetes but actually not diabetes while TN(True Negatives) means actual not diabetes and predicted not diabetes.

\subsection{CORRELATION WITH THE OUTCOMES}

In this study, the correlation between all attributes is computed using the approach discussed in subsection 3.3 and displayed in figure 4 for the values of the correlation coefficients between independent attributes and the dependent class variable. The correlation threshold used is 0.625 , and features with less than 0.625 correlation are eliminated from the training dataset.

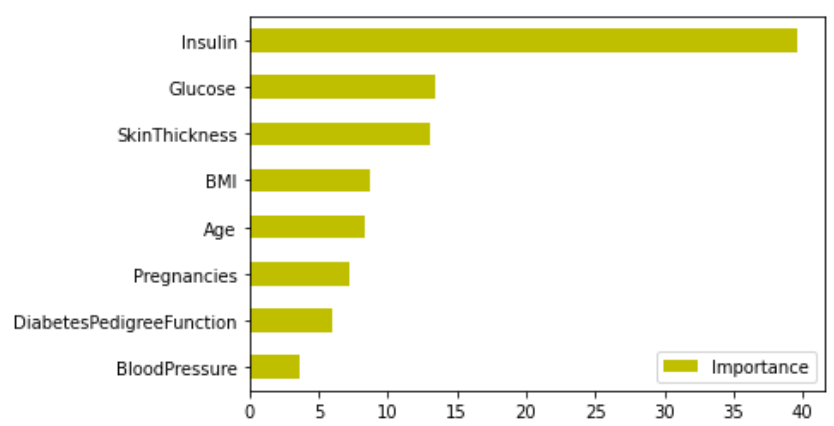

Fig. 4: Attribute's correlation with the outcome

\subsection{Comparison With Different OUtcomes}

Figure 5 shows the prediction results obtained from confusion matrix for all methods. The results show that RF predicts the most TP and NB predicts the most FN. Though RF trees were increased exactly like DT, yet it has highest TP. This is because the approach of data division was handle differently compared to DT as RF only divides the data set into sub-samples and then uses averaging to fit it on the sub-sample DT classifiers. In addition, feature selection is the justification for the overall improvement for all the algorithms especially in TP.

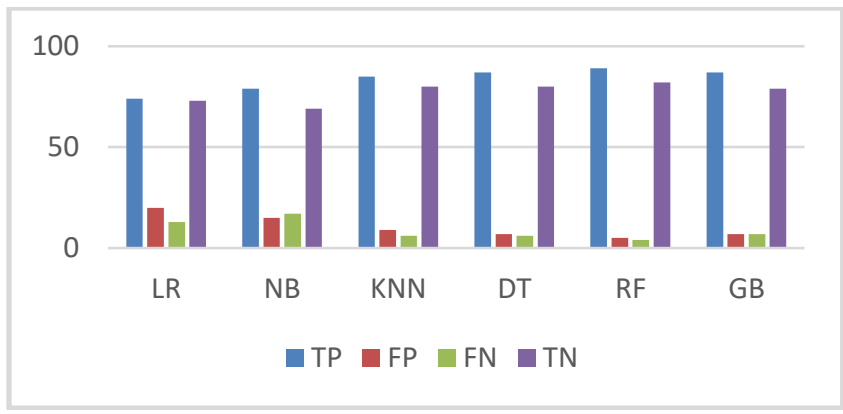

Fig. 5: Comparison of TP, FP, FN and TN on classifiers

\subsection{Comparison Of MOdels BASEd ON MEtrics}

Figure 6 and Table 4 show that LR beats all other machine learning algorithms, with a maximum accuracy of $95 \%$, while DT came in second $(92.78 \%)$. Furthermore, RF (94.25\% and $95.35 \%)$ and DT (91.95\% and 93.02\%) have exhibited the highest precision and recall respectively. RF has the maximum recall of $95.35 \%$, indicating that it is the best classifier for identifying patients with the diabetes diseases. In addition, the work recorded high values in the three metrics can be justified that input fed into algorithms are balance, while the approach was also strengthened with the method of the feature selection.

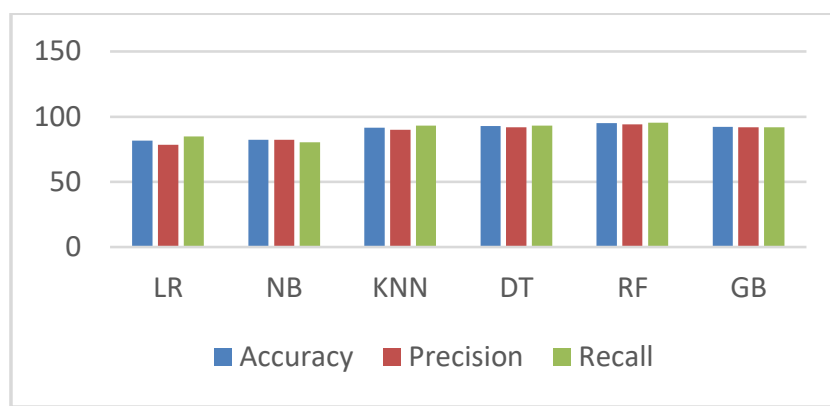

Fig. 6: Accuracy, Precision and Recall on classifiers

Table 4. Performance Evaluation on Six Techniques

\begin{tabular}{cccc}
\hline Models & Accuracy & Precision & Recall \\
\hline LR & 81.67 & 78.49 & 84.88 \\
NB & 82.22 & 82.14 & 80.23 \\
KNN & 91.67 & 89.89 & 93.02 \\
DT & 92.78 & 91.95 & 93.02 \\
RF & 95 & 94.25 & 95.35 \\
GB & 92.2 & 91.86 & 91.86 \\
\hline
\end{tabular}

\section{ConCLUSION}

Diabetes disease prediction on PID dataset has been improved on LR, NB, KNN, DT, RF and GB using the strength of processing techniques. Addressing the problem of missing values, rebalancing the dataset and feature selection method play critical role in robust and precise prediction, as evidenced from the research. The skewness of the attribute distribution was improved with median imputation while the oversampling and undersampling assist in the balancing the dataset. The Pearson correlation feature selection also help to determine the high-risk attributes. As a result, the suggested methodologies will aid medical specialists in making the 
best decision possible in the face of limited knowledge, lowering total treatment costs and improving quality of life. This work has some limitations, as only Pearson correlation method is used for selecting features. In the future, this work can be explored by hybridizing some of the algorithms and testing on mean imputation to see the variance in the performance.

\section{REFERENCES}

American Diabetes Association. (2014). Diagnosis and Classification of Diabetes Mellitus. Diabetes Care, 37(1) 82-90

Azad, C., Bhushan, B., Sharma, R., Shankar, A., Singh, K. K., and Khamparia, A. (2021). Prediction model using SMOTE, genetic algorithm and decision tree for classification of diabetes mellitus. Multimedia Systems, 1-19

Blagus, R. and Lusa, L. (2015). Joint use of over- and under-sampling techniques and cross-validation for the development and assessment of prediction models. BMC Bioinformatics, 16(1) 210

Blake, C. L. and Merz, C. J. (1998). UCI Repository of Machine Learning Databases. Department of Information and Computer Science, University of California, Irvine, CA, USA. Retrived from http://www.ics.uci. edu/mlearn/MLRepository.html on $10 / 4 / 2017$

Boughorbel, S., Jarray, F., and El-Anbari, M. (2017). Optimal classifier for imbalanced data using Matthews Correlation Coefficient metric. PLOS ONE, 12(6), 1-17

Bron, E. E., Smits, M., Niessen, W. J. and Klein, S. (2015). Feature Selection Based on the SVM Weight Vector for Classification of Dementia. IEEE Journal of Biomedical and Health Informatics, 19(5) 1617-1626

Cho, N. H., Shaw, J. E., Karuranga, S., Huang, Y., Rocha F. J. D., Ohlrogge, A. W. and Malanda, B. (2018). IDF Diabetes Atlas: Global estimates of diabetes prevalence for 2017 and projections for 2045. Diabetes Research and Clinical Practice, 138: 271-281

Cover, T. M. (1965). Geometrical and Statistical Properties of Systems of Linear Inequalities with Applications in Pattern Recognition. IEEE Transactions on Electronic Computers, (3), 326-334

Danaei, G., Finucane, M. M., Lu, Y., Singh, G. M., Cowan, M. J., Paciorek, C. J., Lin, J. K., Farzadfar, F., Khang, Y.H., Stevens, G. A., Rao, M., Ali, M. K., Riley, L. M., Robinson, C. A. and Ezzati, M. (2011). National, regional and global trends in fasting plasma glucose and diabetes prevalence since 1980: Systematic analysis of health examination surveys and epidemiological studies with 370 country-years and 2.7 million participants. The Lancet, 378: $31-40$

Daru, A. F., Hanif, M. B. and Widodo, E. (2021). Improving Neural Network Performance with Feature Selection Using Pearson Correlation Method for Diabetes Disease Detection. Jurnal Informatika, 9(1) 123-130

Hasan, M. K., Alam, M. A., Das, D., Hossain, E. and Hasan, M. (2020). Diabetes Prediction Using Ensembling of Different Machine Learning Classifiers. IEEE Access, 8:76516-76531.

Iancu, I., Mota, M. and Iancu, E. (2008). Method for the analysing of blood glucose dynamics in diabetes mellitus patients. IEEE International Conference on Automation, Quality and Testing Robotics, 60-65.

Jeatrakul, P., Wong, K. W., and Fung, C. C. (2010). Classification of Imbalanced Data by Combining the Complementary Neural Network and SMOTE Algorithm.. Models and Applications, 6444, 152-159

Kalaiselvi, K. and Sujarani, P. (2018). Correlation Feature Selection (CFS) and Probabilistic Neural Network (PNN) for Diabetes Disease Prediction. International Journal of Engineering and Technology, 7(3.27) 325-330

kumari, M., and Ahlawat, P. (2021). DCPM: An effective and robust approach for diabetes classification and prediction. International
Journal of Information Technology, 13(3) 1079-1088

Kumari, M., Singh, V. and Ahlawat, P. (2020). Automated Decision Support System for Breast Cancer Prediction. International Journal on Emerging Technologies, 11:193-201

Mahedy, S. M., Rabbi, M. F., Champa, A. I. and Zaman, M. A. (2020). An Effective Diabetes Prediction System Using Machine Learning Techniques. 2020. 2nd International Conference on Advanced Information and Communication Technology, 23-28

Maniruzzaman, M., Kumar, N., Menhazul A, M., Shaykhul I. M., Suri, H. S., El-Baz, A. S. and Suri, J. S. (2017). Comparative approaches for classification of diabetes mellitus data: Machine learning paradigm. Computer Methods and Programs in Biomedicine, 152:23-34.

Maniruzzaman, M., Rahman, M. J., Ahammed, B., and Abedin, M. M. (2020). Classification and prediction of diabetes disease using machine learning paradigm. Health Information Science and Systems, 8(1), 1-14

Nnamoko, N., and Korkontzelos, I. (2020). Efficient treatment of outliers and class imbalance for diabetes prediction. Artificial Intelligence in Medicine, 104: 101815

Ogedengbe, M. T. and Egbunu, C. O. (2020). CSE-DT Features Selection Technique for Diabetes Classification. Applications of modelling and simulation, 4:101-109

Schober, P., Boer, C. and Schwarte, L. A. (2018). Correlation Coefficients: Appropriate Use and Interpretation. Anesthesia and Analgesia, 126(5) 1763-1768

Shafi, S., and Ansari, G. A. (2021). Early Prediction of Diabetes Disease and amp; Classification of Algorithms Using Machine Learning Approach. SSRN Electronic Journal, 1-10

Sreejith, S., Khanna Nehemiah, H., and Kannan, A. (2020). Clinical data classification using an enhanced SMOTE and chaotic evolutionary feature selection. Computers in Biology and Medicine, 126:103991

Tsang, H. C., Chih, P. W. and Tseng, V. S. (2006). Feature Selection for Medical Data Mining: Comparisons of Expert Judgment and Automatic Approaches. 19th IEEE Symposium on ComputerBased Medical Systems, 165-170

Vaishali, R., Sasikala, R., Ramasubbareddy, S., Remya, S., and Nalluri, S. (2017). Genetic algorithm based feature selection and MOE Fuzzy classification algorithm on Pima Indians Diabetes dataset. International Conference on Computing Networking and Informatics, 15:1-5

Yusuf, A., and Kiyea, C. (2021). Prediction of Heart Disease Using Machine Learning Algorithms. Journal of Science Technology and Education, 9(2) 392-405 